\title{
In silico determination of surface entropy of 1-D copper nanostructures
}

\author{
A. Dutta $\cdot$ M. Bhattacharya $\cdot$ N. Gayathri . \\ G. C. Das $\cdot$ P. Barat
}

Received: 31 October 2011/Accepted: 16 February 2012/Published online: 6 March 2012

(C) The Author(s) 2012. This article is published with open access at Springerlink.com

\begin{abstract}
Metallic one-dimensional (1-D) nanostructures are widely studied owing to their important role in developing electronic and electromechanical systems at the nanoscale. In the context of their structures, the large surfaces play a governing role in dictating many of their fundamental characteristics and hence, the surface properties are the most vividly studied issues. In the present work, we employ the harmonic oscillator model to analyze the thermodynamic properties of 1-D copper nanostructures. Our simulations reveal that owing to the large surface to volume ratio, the structural energies of these nanomaterials significantly exceed that of the bulk copper. Nevertheless, the harmonic oscillator approach enables us to directly evaluate the free energy of the system and eventually provides the associated entropy. The calculations are performed for three different crystal orientations and the results clearly indicate that the per atom entropy of thinner nanostructures is larger than their bulk counterpart. This increment in entropy is attributable to the increased degrees of freedom of the surface atoms and has the tendency of stabilizing the surface structure. The harmonic oscillator model works over a reasonable range of temperature and the technique demonstrated here is well extendable to other nanomaterials of interest.
\end{abstract}

Keywords 1-D Nanostructure - Atomistic simulation . Harmonic oscillator · Surface entropy

\footnotetext{
A. Dutta $(\bowtie)$ - G. C. Das

Department of Metallurgical and Materials Engineering, Jadavpur University, Kolkata 700 032, India

e-mail: amlandutta2003@gmail.com

M. Bhattacharya · N. Gayathri · P. Barat Variable Energy Cyclotron Centre, 1/AF Bidhan Nagar, Kolkata 700 064, India
}

\section{Introduction}

Over the past several years, there has been an overwhelming increase in research activities in the field of onedimensional (1-D) metallic nanostructures. This is on account of their extensive applications in nanoelectronic devices (Nakamura et al. 1999; Gelves et al. 2006; He et al. 2008) and nanoelectromechanical systems (Fan et al. 2005; $\mathrm{Li}$ et al. 2007) in the form of nanorods, nanotubes, nanobeams, nanocantilevers and nanowires. In this context, copper became a widely studied material because of its excellent properties and easy availability at low cost. In particular, advents of the in silico methods have boosted much research in this direction. These copper nanostructures are found to exhibit interesting elastic (Liang and Zhou 2003; Liang et al. 2005) as well as plastic (Park et al. 2006; Ji and Park 2007; Sutrakar and Mahapatra 2008) properties. Besides, they posses peculiar features regarding structural transformations (Guo and Guo 2006; Kang and Hwang 2003), ballistic transport (He et al. 2008), melting (Hwang and Kang 2003; Zhang and He 2010), vibration (Kang and Hwang 2003) and point defects (Kang et al. 2002; Onat et al. 2009).

In the case of 1-D nanostructures, the large surface to volume ratio makes the surface effects extremely prominent. The large exposed surfaces are highly energetic and often undergo spontaneous relaxations, thereby reducing the surface energies (Aghemenlo et al. 2006; Crowson et al. 2007; Wu 2009). This manifests in an altered value of the interplanar spacing at the surface layers, both normal and parallel to the plane of the surface (Sokolov et al. 1984; Sinnott et al. 1991; Matveev 2008). In addition, a significant surface stress is created, which can even induce elastic nonlinearity in the 1-D systems (Liang et al. 2005). Most of the studies on surface relaxation deal with the analysis and

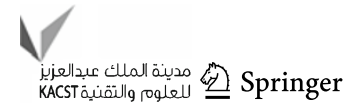


computation of surface energies and the energies of relaxation (Diao et al. 2004; Ouyang et al. 2008; Lu et al. 2008). A large fraction of such results are the outcome of first principle calculations and classical atomistic simulations.

The work reported in this communication is based upon the idea that the nanoscale devices are actually meant to be used at finite temperatures. Therefore, the energies of surface (computable from direct molecular statics simulation) alone would not disclose the true picture of the underlying thermodynamics. Instead, the entropic contributions must also be considered with equal weight to have a broader perspective of this process. Here, we perform the atomistic simulation of surface relaxation of 1-D copper nanostructures of different sizes and compute the free energies and entropies corresponding to this phenomenon. The harmonic oscillator model has been employed for the purpose of free-energy calculations.

\section{Thermodynamic model}

Consider an ensemble of $N$ strongly interacting particles, each of mass $m$, with $3 N$ degrees of freedom. The Hamiltonian of this ensemble can be written as

$H(\mathbf{r}, \mathbf{p})=\frac{|\mathbf{p}|^{2}}{2 m}+V(\mathbf{r})$,

where the vectors $\mathbf{r}$ and $\mathbf{p}$ denote the position and momentum of the system in the phase space, and $V$ is the potential function of the system. If the system is in a local potential minimum $V_{0}$, the Hamiltonian in the close neighborhood of the point in the phase space at equilibrium is given by

$H(\mathbf{r}, \mathbf{p})=V_{0}+\frac{1}{2} \delta \mathbf{r}^{T} \cdot \Im . \delta \mathbf{r}+\frac{|\mathbf{p}|^{2}}{2 m}$,

where $\Im=\partial^{2} V(r)$ is the Hessian matrix with $3 N \times 3 N$ components and $\delta \mathbf{r}$ is a small displacement vector around the equilibrium. The Hessian matrix, being symmetric, yields a real matrix $\Omega$ upon diagonalization. Orthogonal transformation of both sides of Eq. 2 yields

$H\left(\mathbf{r}^{\prime}, \mathbf{p}^{\prime}\right)=V_{0}+\frac{1}{2} \sum_{i=1}^{3 N} \Omega_{i} \cdot\left\langle\delta r_{i}^{\prime 2}\right\rangle+\frac{\left|\mathbf{p}^{\prime}\right|^{2}}{2 m}$.

Here $\Omega_{i}$ denotes the $i$ th element of the matrix $\Omega$ and the prime $\left({ }^{\prime}\right)$ indicates the transformed values. We observe that Eq. 3 resembles the Hamiltonian of an ensemble of $3 N$ non-interacting harmonic oscillators with spring constants $\Omega_{i}$. Therefore, the eigenfrequencies corresponding to these oscillators are $\omega_{i}=\left(\Omega_{i} / m\right)^{1 / 2}$. Once the eigenfrequencies are estimated, we can compute the total free energy of the system as
$F=V_{0}+k T \sum_{i=1}^{3 N} \ln \frac{\hbar \omega_{i}}{k T}$

where $\hbar$ and $k$ are the Planck's constant and the Boltzmann's constant, respectively.

The most advantageous feature of this model is its simplicity itself. The only major atomistic computations required are the structural relaxation of the ensemble, followed by the construction of the Hessian matrix. Nevertheless, one primary source of inaccuracy appears in Eqs. 2 and 3, where the cubic and higher order terms are ignored. Thus, the outcome of this implementation is expected to be reliable as long as the studied temperature range is low to moderate. At higher temperatures, nonlinear effects can predominate and the methodology may become erroneous. For such circumstances, the techniques of thermodynamic integration (de Koning et al. 2000) can be more preferable. So far, various versions of the harmonic oscillator model have been applied to a variety of problems ranging from the dislocation vacancy interaction (Bulatov and Cai 2006) to the estimation of free energies of biomolecules (Yoshioki 2004).

\section{Atomistic simulation}

All the simulations described in this paper have been performed using the MD++ molecular dynamics code (http://micro.stanford.edu). We simulate bulk and 1-D copper nanostructures with the many body embedded-atom model (EAM) potential. The EAM potential has already been discussed widely in different literatures. As the potential satisfactorily reproduces the elastic properties and the energetics of defects and surfaces, it has been commonly used in the classical atomistic computations for similar studies.

By the term 1-D nanostructure, we denote a system of nanoscale size, with its lateral dimensions much smaller than its longitudinal dimension. We study single crystalline structures of square and rectangular cross sections. Examples of earlier studies of similar systems can be found in many of the given references. Three types of crystal structures are used as described below.

Structure 1. The [001] is the longitudinal direction while the (100) and (010) facets are the exposed surfaces. The cross section is square.

Structure 2. The [001] direction is longitudinal and (110), (110) planes are at the free surfaces of the system with square cross section.

Structure 3. The system is oriented along $1 \overline{2} 1$ and (101), (111) are the free surfaces. It has a rectangular cross section with aspect ratio $\sim 1.225$. 
A typical simulation cell is shown in Fig. 1. The cell is periodic only along the longitudinal direction $(x)$, effectively making the system infinitely long but finite in the other two directions. Because of 1-D periodicity, it is sufficient to simulate with only a few atoms along the longitudinal direction, which is of particular advantage in terms of computational load.

Before determining the Hessian matrix, it is essential to minimize the system to its local potential minimum. A straightforward structural relaxation of the simulated system can generate a flawed matrix. This happens when the algorithm reduces the potential energy, but fails to converge to the exact potential minimum. In some other cases, the algorithm converges to the nearest shallow local minimum and misses a nearby state which can be in a deeper potential well. Such conditions can be detected by inspecting the eigenvalues. In principle, the first three eigenvalues must be zero as they correspond to the translation of the entire crystal, whereas the rest of them should be positive definite. Nevertheless, we have observed that imperfect relaxation may manifest in small negative eigenvalues. Clearly, direct relaxation if the structure is inefficient as the algorithm would generate the same result at each implementation. In our work, we circumvent this problem by allowing the system to explore the phase space dynamically. This is implemented through a series of molecular dynamics simulations at different temperatures. With random choice of initial atomic velocities, equilibration runs are carried out in the sequence of 150, 100, 50, 10 and $5 \mathrm{~K}$ at a small time step of $0.5 \mathrm{fs}$. Each run is performed for 1,000 time steps, followed by structural relaxation using the conjugate gradient algorithm. During the relaxation process, dimension of the simulation cell is allowed to vary longitudinally so that the residual stresses

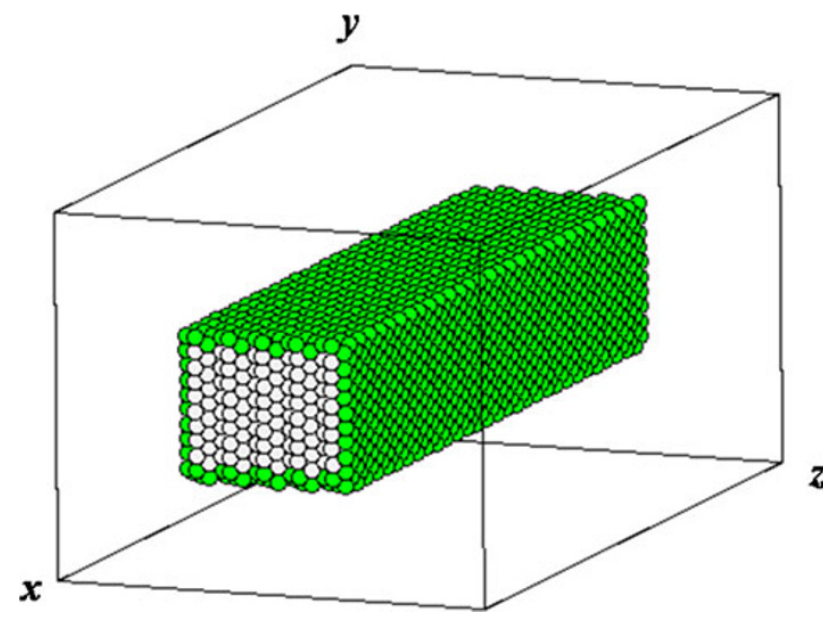

Fig. 1 A typical simulation cell periodic along the $x$ direction. The surface atoms are separately shown in green and are selected by energy filtering
(Liang et al. 2005; Wu 2009) can be minimized. Because of random initial conditions, the thermal fluctuation is now capable of knocking the structure out of an inappropriate potential valley. We have observed this method to enhance the probability of perfect energy minimization to a significant extent. Once the relaxed structure has been obtained, we can easily generate the Hessian matrix and calculate the free energies as described in the previous section.

\section{Results and discussion}

The free energy versus temperature plot is shown in Fig. 2. This result corresponds to an ensemble of 192 atoms in the bulk crystal simulated with periodic boundary conditions in all the three directions. This trend is typical of all such freeenergy plots, where the energy maxima are obtained because of the inherent feature of Eq. 4. However, from the perspective of physical implication, the free-energy differences yield more meaningful information than the absolute free-energy values and hence all the results obtained for the nanostructures are compared with that of the bulk. Moreover, while comparing the thermodynamic quantities such as changes in the internal energy, free energy and entropy for different lateral dimensions of the nanostructures, it is preferable to express them as average values per atom, since these quantities are extensive in nature.

Figure 3 displays the potential energy differences $\Delta E$ ( $E_{\text {nano }}-E_{\text {bulk }} /$ atom $)$ between the relaxed nanostructures and the bulk for the three configurations under study. We observe that although the surface relaxation compensates for the large surface energies, the system is still at a

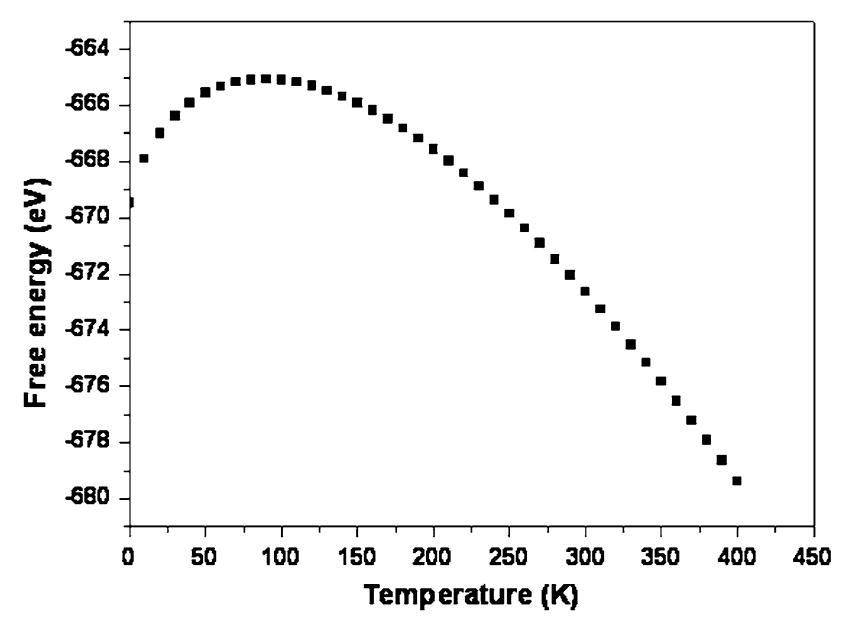

Fig. 2 Free energy versus temperature plot for the bulk copper. The negative values are the result of the large cohesive energy of the metal

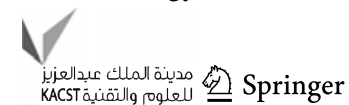




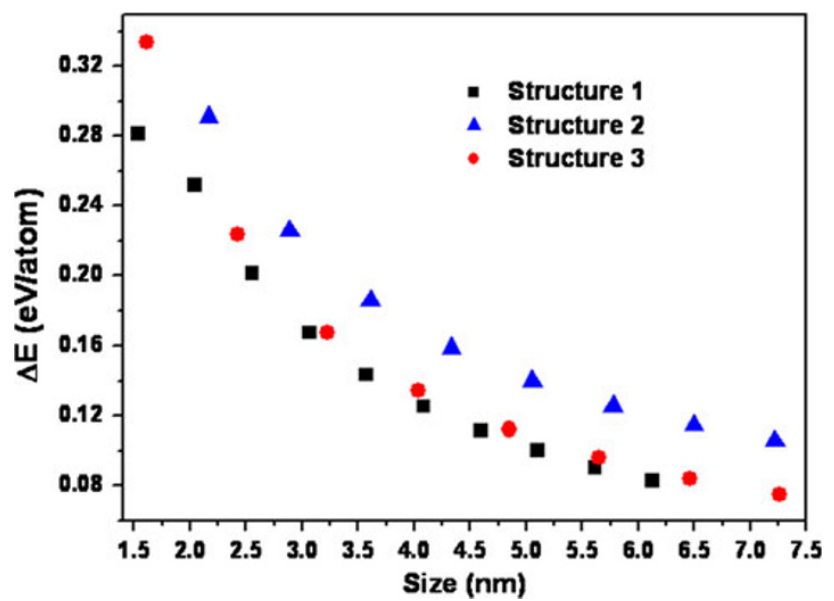

Fig. 3 The structural potential energies $(\Delta E /$ atom) of the nanostructures with variation in system size. The square, triangular and circular marks represent the values for structures 1, 2 and 3, respectively. Clearly, structure 2 is most energetic out of all the three studied systems, owing to its large surface energy. Moreover, the potential energies increases with the reduction in size due to enhanced surface to volume ratio

higher energy state as compared to the bulk. With increment in the size of the structure, the energy decreases and approaches that of the bulk. As $\Delta E$ is larger for the structure 2 as compared to the other systems, the relaxation brings the structures 1 and 3 to states that are apparently more stable than the structure 2 .

Next we study the entropies of the 1-D nanostructures. For this purpose, the free-energy differences $\Delta F\left(F_{\text {nano }}-\right.$ $F_{\text {bulk }} /$ atom) of the three systems at $300 \mathrm{~K}$ are evaluated using the Eq. 4. These free-energy differences involve the change in the structural energy of the system as well as the change in the entropies due to creation of free surfaces, followed by surface relaxation. Once the free energies have been computed, the entropic contributions can be estimated as $\Delta S=(\Delta E-\Delta F) / T$. An important concept is worthy of being discussed at this point. The internal energy difference $\Delta E$ can be split into static and thermal components as $\Delta E=\Delta E_{\text {stat }}+\Delta E_{\text {therm. }}$. We measure $\Delta F$ and $\Delta S$ at a finite temperature $T$, whereas $\Delta E$ is obtained from the static ( $T=0 \mathrm{~K}$ ) simulations. The use of the static value of $\Delta E$ in calculating $\Delta S$ at non-zero temperature does not produce an error (Bulatov and Cai 2006), for the average thermal energy of a system $\left(E_{\text {therm }}\right)$ with harmonic potential is proportional to the temperature $T$. Consequently, the thermal contributions cancel out while calculating $\Delta E / T$ and the potential function $V(\mathbf{r})$ dictates the quantity alone. Figure 4 displays the entropy changes at $300 \mathrm{~K}$. The entropic benefits are clearly more for the smaller nanostructures. Interestingly, the entropy change $(\Delta S /$ atom) is maximum for the structure 2 , which implies the largest entropic stability despite its maximum instability in terms of the corresponding $\Delta E$ value as exhibited in Fig. 3.

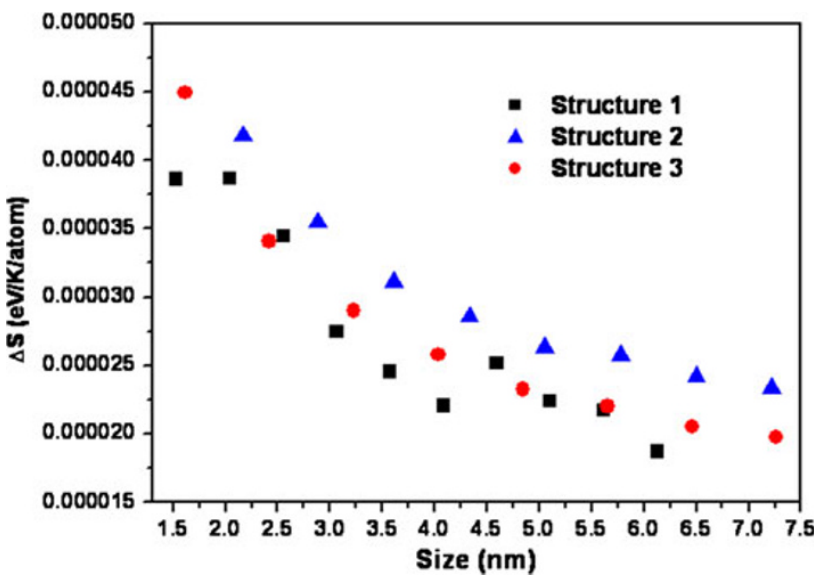

Fig. 4 The change in entropy ( $\Delta S /$ atom) of the 1-D nanostructures, as the functions of system size for the studied structures. Structure 2 can be seen to avail the maximum entropic benefit. The gain in the entropies is larger for the smallest systems and reduces with increase in the system size

Another important observation in this regard is the enhanced entropy for the thinner nanostructures. This size effect can be attributed to the larger exposed surface in smaller nanosystems. Owing to their reduced coordination numbers, the boundary atoms are loosely bound, which manifests in the increased effective degrees of freedom and. Consequently, the per atom entropy rises as the size of the 1-D nanostructure reduces (Fig. 4).

We have presented all the results of computation at $300 \mathrm{~K}$. The influence of the ambient temperature on the thermodynamic quantities can be estimated directly without separate calculations. Consider two arbitrary configurations, say $\mathrm{C}$ and $\mathrm{C}^{\prime}$, with the same number of atoms and the interatomic potential function. If $\left\{\omega_{i}\right\}$ and $\left\{\omega_{i}^{\prime}\right\}$ be their respective eigenfrequencies, from Eq. 4, the free-energy difference varies with the temperature linearly as

$\Delta F(T)=\Delta E+k T \ln \prod_{i=1}^{3 N} \frac{\omega_{i}}{\omega_{i}^{\prime}}$.

Similarly, the entropy change is given as

$\Delta S=k \ln \prod_{i=1}^{3 N} \frac{\omega_{i}^{\prime}}{\omega_{i}}$

Equation 6 indicates that in general, the harmonic oscillator model predicts that the entropic variation of a system is independent of the temperature and solely depends on the equilibrium atomic configuration. As a result, the entropic stability contribution $T \Delta S$ becomes a linear function of the system's temperature. However, it must be noted that in cases where the temperature is too large, or when the interatomic potential is highly nonlinear or soft, the abovementioned conclusion may not remain valid and $\Delta S$ can become a function of the temperature. 


\section{Conclusion}

By the way of summary, we have studied the thermodynamic quantities associated with the surface relaxation of one-dimensional copper nanostructures. The study reveals that the process of creating free surface is capable of inducing entropic stability to a nanostructure. The method of free-energy calculation using the harmonic oscillator model has been demonstrated. The present work can be considered as a case study and the methodology can be extended to other systems of interest. We reveal that the analysis of the underlying thermodynamics on the basis of structural energy is incomplete and must be supplemented with the entropic contributions to arrive at the final quantitative predictions.

Acknowledgments One of the authors (AD) acknowledges the financial support provided by the Council of Scientific and Industrial Research, India, in the form of senior research fellowship.

Open Access This article is distributed under the terms of the Creative Commons Attribution License which permits any use, distribution, and reproduction in any medium, provided the original author(s) and the source are credited.

\section{References}

Aghemenlo HE, Iyayi SE, Avwiri GO (2006) Surface relaxation and surface energy of face-centered cubic metals. J Appl Sci Environ Manage 10:37-42

Bulatov VV, Cai W (2006) Computer simulations of dislocations. Oxford University Press, New York

Crowson DA, Farkas D, Corcoran SG (2007) Geometric relaxation of nanoporous metals: the role of surface relaxation. Script Matter 56:919-922

de Koning M, Cai W, Antonelli A, Yip S (2000) Efficient free-energy calculations by the simulation of nonequilibrium processes. Comput Sci Eng 2:88-96

Diao J, Gall K, Dunn ML (2004) Atomistic simulation of the structure and elastic properties of gold nanowires. J Mech Phys Solids 52:1935-1962

Fan DL, Zhu FQ, Cammarata RC, Chien CL (2005) Controllable high-speed rotation of nanowires. Phys Rev Lett 94:247208

Gelves GA, Lin B, Sundararaj U, Haber JA (2006) Low electrical percolation threshold of silver and copper nanowires in polystyrene composites. Adv Funct Mater 16:2423-2430
Guo Y, Guo W (2006) Structural transformation of partially confined copper nanowires inside defected carbon nanotubes. Nanotechnology 17:4726-4730

He C, Zhang P, Zhu YF, Jiang Q (2008) Structures and quantum conduction of copper nanowires under electric fields using first principles. J Phys Chem C 112:9045-9049

Hwang HJ, Kang JW (2003) Melting and breaking of ultrathin copper nanobridges. Surf Sci 532-535:536-543

Ji C, Park HS (2007) Characterizing the elasticity of hollow metal nanowires. Nanotecnology 18:115707

Kang JW, Hwang HJ (2003) Molecular dynamics simulations of ultrathin $\mathrm{Cu}$ nanowires. Comput Mater Sci 27:305-312

Kang JW, Seo JJ, Byun KR, Hwang HJ (2002) Defects in ultrathin copper nanowires: atomistic simulations. Phys Rev B 66:125405

Li M, Mayer TS, Sioss JA, Keating CD, Bhiladvala RB (2007) Template-grown metal nanowires as resonators: performance and characterization of dissipative and elastic properties. Nano Lett 7:3281-3284

Liang W, Zhou M (2003) Response of copper nanowires in dynamic tensile deformation. Proc Inst Mech Eng Part C 218:599-606

Liang H, Upmanyu M, Huang H (2005) Size-dependent elasticity of nanowires: nonlinear effects. Phys Rev B 71:241403(R)

Lu HM, Han FQ, Meng XK (2008) Size-dependent thermodynamic properties of metallic nanowires. J Phys Chem B 112:9444-9448

Matveev AV (2008) Calculation of the lattice relaxation of metallic surfaces in terms of the density-functional method. Phys Met Metallogr 105:427-434

Nakamura A, Brandbyge M, Hansen LB, Jacobsen KW (1999) Density functional simulation of a breaking nanowire. Phys Rev Lett 82:1538-1541

Onat B, Konuk M, Durukanoğlu S, Dereli G (2009) Energetics and atomic relaxations of $\mathrm{Cu}$ nanowires: the effect of local strain and cross-sectional area. Nanotecnology 20:075707

Ouyang G, Li XL, Tan X, Yang GW (2008) Surface free energy of nanowires. Nanotechnology 19:045709

Park HS, Gall K, Zimmerman JA (2006) Deformation of FCC nanowires by twinning and slip. J Mech Phys Solids 54:1862-1881

Sinnott SB, Stave MS, Raeker TJ, DePristo AE (1991) Corrected effective-medium study of metal-surface relaxation. Phys Rev B 44:8927-8941

Sokolov J, Jona F, Marcus PM (1984) Trends in metal surface relaxation. Solid State Commun 49:307-312

Sutrakar VK, Mahapatra DR (2008) Formation of stable ultra-thin pentagon $\mathrm{Cu}$ nanowires under high strain rate loading. J Phys Condens Matter 20:335206

Wu HA (2009) Molecular dynamics study on mechanics of metal nanowires. Mech Res Commun 33:9-16

Yoshioki S (2004) Modelling free energy of a rigid protein in solid water: comparison between rigid-body motions and harmonic oscillators. Phys A Stat Mech Appl 331:552-570

Zhang WX, He C (2010) Melting of $\mathrm{Cu}$ nanowires: a study using molecular dynamics simulation. J Phys Chem C 114:8717-8720 